IIIIIIIIIIIIIIIIIIIIIIIIIIIIIIIIIII

Original Article

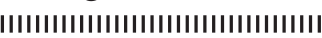

\title{
Biological activity and disease controlling efficacy of penthiopyrad
}

\author{
Yuji Yanase, ${ }^{1, *}$ Junro Kishi, ${ }^{2}$ Syunichi Inami, ${ }^{3}$ Hiroyuki Katsuta $^{4}$ \\ and Yukihiro Yoshikawa ${ }^{4}$ \\ ${ }^{1}$ Research \& Development Division, Mitsui Chemicals Agro, Inc., Shiodome City Center, \\ 1-5-2 Higashi-Shimbashi, Minato-ku, Tokyo 105-7117, Japan \\ ${ }^{2}$ Sales \& Marketing Division, Mitsui Chemicals Agro, Inc., Shiodome City Center, \\ 1-5-2 Higashi-Shimbashi, Minato-ku, Tokyo 105-7117, Japan \\ ${ }^{3}$ Agrochemicals Research Institute, Mitsui Chemicals Agro, Inc., 1358 Ichimiyake, Yasu, Shiga 520-2342, Japan \\ ${ }^{4}$ Mobara Research \& Development Center, Mitsui Chemicals, Inc., 1144 Togo, Mobara, Chiba 297-0017, Japan
}

(Received October 22, 2012; Accepted August 15, 2013)

\begin{abstract}
Various carboxamide derivatives were synthesized, and the fungicidal activity was evaluated by both in vitro and in vivo experiments to find out the best compound for a fungicide. Penthiopyrad is unique in its wide fungicidal spectrum. The introduction of a thiophene ring with hydrophobic branched alkyl groups brought broad-spectrum fungicidal activity leading to the discovery of penthiopyrad, $N$-[2-(1,3-dimethylbutyl)-3-thienyl]-1-methyl-3-(trifluoromethyl)-1H-pyrazole-4-carboxamide. Of the carboxamide fungicides, carboxin is particularly effective for controlling the diseases caused by basidiomycetous fungi, including smut and bunt of cereals, but it is not as effective against others that are easily controlled by boscalid, which is not so effective against basidiomycetes pathogens. Penthiopyrad covers the effect gap between carboxin and boscalid. In this report, we have focused on the biological properties revealed in laboratory tests and small pot tests in the greenhouse. (๑) Pesticide Science Society of Japan

Keywords: penthiopyrad, carboxamide, fungicide, biological properties.
\end{abstract}

\section{Introduction}

As the first generation compound of carboxamide, carboxin (1) was developed more than 40 years ago and has been used as an important seed treatment fungicide. ${ }^{1)}$ Mepronil and flutolanil, developed in the 1980s, have been also used to control some diseases caused by basidiomycetes such as Thanatephorus cucumeris (ana. Rhizoctonia solani). Then furametpyr and thifluzamide were developed in the late 1990s and launched to control rice sheath blight caused by $T$. cucumeris, but their spectra were relatively restricted.

On the other hand, there were some precedents suggesting that ortho-substituted carboxanilides could show broader spectrum activity. Edgington reported that some oxathiin compounds showed broader spectra of fungicidal activity. ${ }^{2)}$ For example, $N$-(biphenyl-2-yl)-2-methyl-1,4-thioxolane-3-carboxamide $(\mathrm{F} 427 ; 2)$ has fungicidal activity not only on basidiomycetes but also on ascomycetes. In 1989, Mitsubishi Kasei Corporation (now Nihon Nohyaku Co., Ltd.) discovered a 2-chloropyridine-3-carboxamide derivative, BC723 (3), that exhibited fungicidal activity against the ascomycetous gray mold pathogen

\footnotetext{
* To whom correspondence should be addressed.

E-mail: Yuuji.Yanase@mitsui-chem.co.jp

Published online October 24, 2013

(c) Pesticide Science Society of Japan
}

Botrytis cinerea. ${ }^{3)}$ Additionally, ortho-substituted carboxanilides were of interest as basic structures of a broad spectrum fungicide. In the first test, a mycelium agar disk of $B$. cinerea was used as an inoculum to evaluate the performance of carboxamide, but it resulted in a false and then a novel evaluating system using spore suspension developed to successfully evaluate fungicidal activities in carboxamide compounds. In the course of our research, we paid special attention to the fact that the fungicidal activities of some $\mathrm{N}$-biphenyl carboxamide compounds were moderate but their spectra were broad against various kinds of pathogenic fungi. After extensive research, we found a highly



carboxin (1)

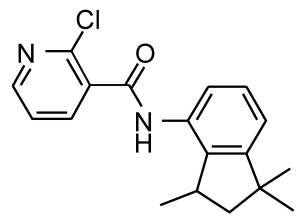

$\mathrm{BC} 723(3)$
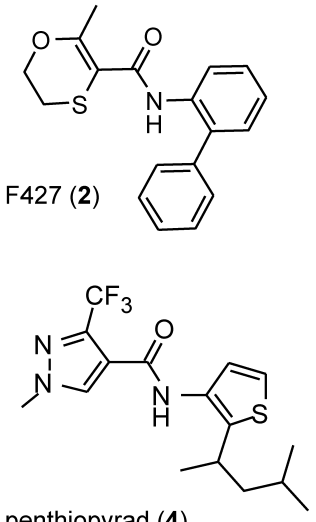

penthiopyrad (4)
Fig. 1. Chemical structure of carboxin-related carboxamides. 
active novel carboxanilide derivative that contained two heteroaromatic rings and also found that branched alkyl substitution on the heteroaromatic ring in the amino part of the carboxanilide expanded its antifungal spectrum. ${ }^{4-8)}$ With further research, we finally discovered penthiopyrad (4), a unique carboxanilide fungicide candidate containing both pyrazole and thiophene rings. ${ }^{7,8)}$

\section{Materials and Methods}

\section{Preparation of test chemicals and fungal materials}

\subsection{Chemicals}

Penthiopyrad was synthesized by Mitsui Chemicals, Inc. A stock solution of penthiopyrad $(5000 \mathrm{mg} / \mathrm{L})$ was prepared in acetone and diluted appropriately for each assay. A 20\% suspension concentrate formulation (Affet ${ }^{\circledR}$ ) of penthiopyrad was also used for in vivo tests. Other commercial fungicides, thiophanate-methyl (Topsin M WP, Nippon Soda Co., Ltd., Tokyo, Japan), procymidone (Sumilex WP, Sumitomo Chemicals, Co., Ltd., Tokyo, Japan), diethofencarb (Powmyl WP, Sumitomo Chemicals, Co., Ltd., Tokyo, Japan), mepanipyrim (Frupica FL, Kumiai Chemical Industry Co., Ltd., Tokyo, Japan) and azoxystrobin (Amister 20 Flowable, Syngenta Japan Co., Ltd., Tokyo, Japan), were purchased and used for pot tests; the active ingredients were extracted from purchased formulations and used for laboratory tests.

\subsection{Fungal materials}

B. cinerea (MCAG stock culture No. 40212), Alternaria mali (MCAG stock culture No. 40103), Sclerotinia sclerotiorum (MCAG stock culture No. 20181), R. solani, and Monilinia mali (MCAG stock culture No. 20121) for mycelial growth inhibition tests were grown and maintained on potato dextrose agar (PDA, Kyokuto Pharmaceutical Industrial Co., Ltd., Tokyo, Japan) plates. Ascospores of S. sclerotiorum for spore germination inhibition test were collected from apothecia grown on sclerotia by the following method. Sclerotia produced on PDA and incubated for 15 to 20 days were harvested and disinfested in $20 \%$ sodium hypochlorite solution for $1 \mathrm{~min}$, then rinsed with sterile water and allowed to dry. Ten to 15 surface disinfested sclerotia were transferred into a sterile pot filled with $40 \mathrm{~mL}$ of dry sand and $10 \mathrm{~mL}$ of deionized water, which was sterilized for $30 \mathrm{~min}$ at $120^{\circ} \mathrm{C}$. The pot was kept at $4^{\circ} \mathrm{C}$ in the dark for 1 month, then placed in a $12^{\circ} \mathrm{C}$ growth chamber in the dark until the stipe was observed. When the stipe grew $5 \mathrm{~mm}$ in length, pots were transferred to a $16^{\circ} \mathrm{C}$ growth chamber with a $12 \mathrm{hr}$ dark and light period until the apothecia were fully matured. Venturia nashicola (MCAG stock culture No. 20024) and Fulvia fulva (MCAG stock culture No. 40331) for spore germination inhibition tests were grown on PDA plates, and conidiospores were collected from the surface of the mycelia. Sphaerotheca cucurbitae (subcultured in greenhouse) and Puccinia recondita (subcultured in growth chamber at $18^{\circ} \mathrm{C}$ ) were maintained on the leaves of cucumber and wheat, respectively, and conidiospores and urediniospores were collected from the diseased leaves just before the tests. The mycelia of $B$. cinerea grown on a PDA plate were illuminated with black light blue lamps (Panasonic FL20S-BL-B) for 3 days to induce spore formation and the spores were collected with a small paintbrush.

Pyricularia oryzae (MCAG stock culture No. 40061), B. cinerea, and $F$. fulva for pot tests of rice blast, kidney bean gray mold, and tomato leaf mold, respectively, were grown on PDA plates to produce conidiospores. Puccinia recondita and Sphaerotheca cucurbitae (subcultured in greenhouse) for pot tests of wheat brown rust and cucumber powdery mildew, respectively, were maintained on the leaves of wheat (Tricicum aestivum cv. Norin No. 61) and cucumber (Cucumis sativa cv. Sagami Hanjiro).

\section{Mycelial growth inhibition tests on agar media}

2.1. Application methods

PDA plates containing penthiopyrad at 250, 50, 10, 2, and $0.4 \mathrm{ppm}$ were prepared. Replication was three for each concentration.

\subsection{Inoculation methods}

B. cinerea, A. mali, S. sclerotiorum, R. solani, and M. mali were previously cultured on PDA plates for 7 days, and mycelial discs were cut off from the fresh part of the colonies by a $6 \mathrm{~mm}$ cork borer. The mycelial discs were put on the PDA plates containing each tested chemical. Controls were incubated on the PDA without any tested chemicals.

\subsection{Assessment methods}

After incubation for 4 days at $20^{\circ} \mathrm{C}$, the colony diameter, with $6 \mathrm{~mm}$ deducted for the inoculated mycelial disc size on agar medium, was measured, and the inhibition rate was calculated by the following formula:

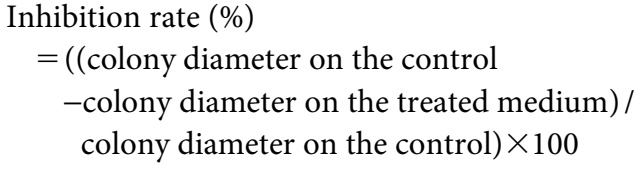

$\mathrm{EC}_{50}$ and minimum inhibitory concentration (MIC) values were calculated by the approximation formula of concentrationeffect curves against each pathogen. The approximation formulas were obtained from the least-square method.

\section{Spore germination inhibition test}

\subsection{Application methods}

PDA solution containing penthiopyrad at 50, 10, 2, and $0.4 \mathrm{ppm}$ was spread on hole glass slides and solidified. Replication was three for each concentration.

3.2. Inoculation methods

Spores collected from the colony were suspended in potato dextrose broth $(\mathrm{PDB})$ to make a spore suspension. Its concentration was adjusted to $1 \times 10^{3}-10^{4}$ spores $/ \mathrm{mL}$, and the spore suspension was dropped onto the surface of the PDA on the hole glass slide and kept in a Petri dish in the dark at $20^{\circ} \mathrm{C}$.

\subsection{Assessment methods}

After incubation for 24 to $48 \mathrm{hr}$, more than 100 spores for each glass slide were observed under a microscope to count the num- 
ber of germinated spores. Inhibition rate was calculated by the following formula:

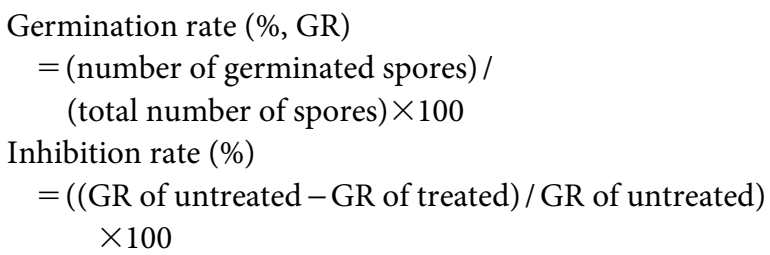

$\mathrm{EC}_{50}$ and MIC values were calculated by the statistical method described in the former assessment method of mycelial growth inhibition tests on agar media.

\section{Pot tests}

\subsection{Preparation of test plants}

After soaking in water for two days at room temperature, about 30 rice (Oryza sativa cv. Tsukimimochi) seeds were sown in a 7.5-cm-diameter plastic pot filled with soil and grown for 10 to 14 days before the inoculation test. About 20 wheat seeds were sown in a 6-cm-diameter pot and grown for 10 to 14-days; a cucumber seed and two kidney bean (Phaseolus vulgaris cv. Greentop) seeds were sown in a 7.5-cm-diameter pot and grown for 10 to 14 days before the inoculation test. Tomato (Solanum lycopersicum cv. Sekaiichi) seeds were sown and grown for 14 days followed by transplanting and incubation in a $7.5-\mathrm{cm}$-diameter pot in a greenhouse before the inoculation test.

\subsection{Application methods}

Fifty milligrams of penthiopyrad was dissolved in $10 \mathrm{~mL}$ of acetone and then diluted with water to adjust the concentration. The compound solution was sprayed on 2- and 3-leaf stage (LS) rice seedlings at 200,100,50, and $12.5 \mathrm{ppm}$; on $1.5 \mathrm{LS}$ wheat seedlings at $100,25,6.25$, and $1.56 \mathrm{ppm}$; on $1.5 \mathrm{LS}$ cucumber seedlings at $100,25,6.25$, and $1.56 \mathrm{ppm}$; on 4 - and 5-LS tomato seedlings at 200,100,50, and $25 \mathrm{ppm}$; and on seed-leaf stage kidney bean seedlings at $125,62.5,31.2,12.5$, and $6.25 \mathrm{ppm}$. Sample water volumes were $30 \mathrm{~mL} / 3$ pots for rice, cucumber, kidney bean, and tomato and $15 \mathrm{~mL} / 3$ pots for wheat. After air drying, plants were inoculated with spores of pathogens by the following methods.

\subsection{Inoculation methods}

\subsubsection{Rice blast}

Treated plants were sprayed with a spore suspension of Pyricularia oryzae adjusted to $1 \times 10^{5}$ spores $/ \mathrm{mL}$ and then kept in a chamber controlled by a 12-hr dark and 12-hr light cycle condition under high humidity at $25^{\circ} \mathrm{C}$ for 7 days.

\subsubsection{Wheat brown rust}

Treated plants were dusted with spores of Puccinia recondita and then kept in a plastic bag to maintain darkness and high humidity at $4^{\circ} \mathrm{C}$ for 2 days followed by incubation in a greenhouse for 8 days.

\subsubsection{Cucumber powdery mildew}

Treated plants were dusted with spores of Sphaerotheca cucurbitae with a paintbrush and incubated in a greenhouse for 7 days.

\subsubsection{Kidney bean gray mold}

Treated kidney bean leaves were cut and put in plastic cups in which humidity was maintained with a wet paper filter. A paper disk (8 mm diameter, thick type; Toyo Roshi Kaisha, Ltd., Tokyo, Japan) dipped in the spore suspension $\left(1 \times 10^{5}\right.$ spores $/ \mathrm{mL}$, containing $20 \%$ potato broth and $2 \%$ glucose) of $B$. cinerea was placed on the center of each cotyledon. The plastic cups were kept in dark conditions at $20^{\circ} \mathrm{C}$ for four days.

\subsubsection{Tomato leaf mold}

Treated tomato plants were sprayed with the spore suspension of F. fulva adjusted to $1 \times 10^{6}$ spores $/ \mathrm{mL}$ and then kept in a greenhouse at $22^{\circ} \mathrm{C}$ for 2 weeks.

4.4. Assessment methods

The lesions and the uredinial colonies on the inoculated leaves were counted in the cases of rice blast and wheat brown rust, respectively, and the disease index was adapted to the following index: 0, no symptoms; 1, 1-3 lesions; 2, 4-7 lesions; 3, 8-14 lesions; and 4, more than 14 lesions. In the cases of cucumber powdery mildew, kidney bean gray mold, and tomato leaf mold, the disease index was calculated by the following index: 0 , no symptoms; 1 , less than $12.5 \%$; 2, 12.5-25.0\%; 3, 25-50\%; and 4, more than $50 \%$ of the leaf area was covered with lesions.

Control efficacy (\%) was calculated by the following formula:

$$
\begin{aligned}
& \text { Disease severity }(\mathrm{DS}) \\
& =\left(\sum_{(4 \times \text { total leaves })}(\text { disease index } \times 100\right. \\
& \text { Control efficacy }(\%) \\
& =((\mathrm{DS} \text { on untreated leaf }-\mathrm{DS} \text { on treated leaf }) / \\
& \quad(\mathrm{DS} \text { on untreated leaf }) \times 100
\end{aligned}
$$

$\mathrm{EC}_{50}$ and minimum inhibitory concentration (MIC) values were calculated by the approximation formula of the concentration-effect curves of each compound against the pathogens. The approximation formulas were obtained from the least-square method.

5. Growth inhibition test for chemical resistant strains of gray mold fungus, B. cinerea, and powdery mildew fungus, Sphaerotheca cucurbitae

5.1. Agar plate dilution test for gray mold fungus, B. cinerea PDA media containing 250, 50, 10, 2, and $0.4 \mathrm{ppm}$ penthiopyrad were prepared. Replication was three for each concentration. As reference compounds of benzimidazoles, dicarboximides, $\mathrm{N}$-phenylcarbamates, and anilinopyrimidines, $100 \mathrm{ppm}$ of thiophanate-methyl, $5 \mathrm{ppm}$ of procymidone, $10 \mathrm{ppm}$ of diethofencarb, and 2 ppm of mepanipyrim were used, respectively. Details of the procedures of application, inoculation, and assessment are given in section 2, "Mycelial growth inhibition tests on agar media."

Five isolates of resistant strains that were classified by the fungicidal activity of thiophanate-methyl, procymidone, and diethofencarb were used for this study. The first isolate is S-MRHR type (MCAG stock culture No. 40214), which shows sus- 
ceptibility to thiophanate-methyl, moderate resistance to procymidone, and high resistance to diethofencarb. The second one is MR-MR-HR type (MCAG stock culture No. 40210), which shows moderate resistance to thiophanate-methyl and procymidone and high resistance to diethofencarb. The third one is HRS-S type (MCAG stock culture No. 40212), which shows high resistance to thiophanate-methyl and susceptibility to procymidone and diethofencarb. The fourth one is HR-MR-WR type (MCAG stock culture No. 40207), which shows high resistance to thiophanate-methyl, moderate resistance to procymidone, and weak resistance to diethofencarb. The fifth one is HR-MRHR type (MCAG stock culture No. 40206), which shows high resistance to thiophanate-methyl and diethofencarb and moderate resistant to procymidone. A strain resistant to anilinopyrimidine compounds that was stocked at the National Institute for Agro-Environmental Sciences was also used.

5.2. Leaf disk test for cucumber powdery mildew fungus, Sphaerotheca cucurbitae

Fifty milligrams of penthiopyrad and azoxystrobin was dissolved in $10 \mathrm{~mL}$ of acetone and diluted with water to adjust to prescribed concentrations. Two strains of Sphaerotheca cucurbitae, susceptible (S-S) and resistant (R-S) to strobilurins, were used. The S-S strain was maintained on the leaves of cucumber in a greenhouse of Mitsui Chemicals Agro, Inc., in Chiba. The R-S strain was isolated from a diseased sample collected in a greenhouse of Mitsui Chemicals Agro, Inc., in Chiba. Leaf disks were prepared from cucumber cotyledon with a $10-\mathrm{mm}$ diameter cork borer and inoculated with conidiospores of the cucumber powdery mildew pathogen and floated on the chemical solutions. Seven days after inoculation, assessment was done by the following index: 0 , no symptoms; 1 , less than $12.5 \% ; 2$, $12.5-25.0 \%$; $3,25-50 \%$; and 4 , more than $50 \%$ of the leaf area was covered with lesions. Control efficacy (\%) was then calculated by the following formula:

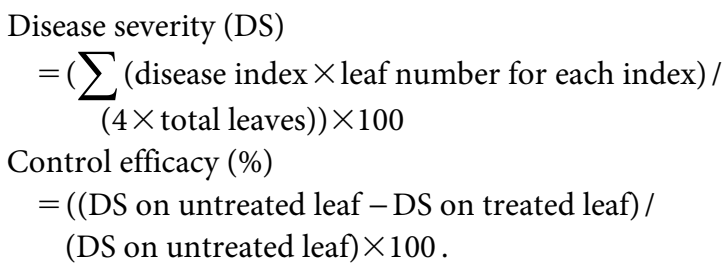

\section{Results and Discussion}

\section{Mycelial growth inhibition on PDA}

Penthiopyrad clearly inhibited the mycelial growth of $A$. mali, M. mali, and Sclerotinia sclerotiorum, but not so in case of $B$. cinerea and $R$. solani on PDA. MIC values of A. mali and Sclerotinia sclerotiorum were less than $10 \mathrm{ppm}$, while they were about $50 \mathrm{ppm}$ and $30 \mathrm{ppm}$ in B. cinerea and R. solani, respectively.

Table 1. Fungicidal activity of penthiopyrad against various pathogens (Mycelial growth inhibition test on agar media)

\begin{tabular}{|c|c|c|c|c|c|c|c|}
\hline \multirow{3}{*}{$\begin{array}{l}\text { Name of } \\
\text { pathogen }\end{array}$} & \multicolumn{5}{|c|}{ \% Inhibition of mycelial growth ${ }^{a}$} & \multirow{3}{*}{$\mathrm{EC}_{50}(\mathrm{ppm})$} & \multirow{3}{*}{$\mathrm{MIC}(\mathrm{ppm})$} \\
\hline & \multicolumn{5}{|c|}{ Concentration of penthiopyrad (ppm) } & & \\
\hline & 250 & 50 & 10 & 2 & 0.4 & & \\
\hline A. mali & 100 & 100 & 100 & 96 & 73 & $<0.4$ & 6.6 \\
\hline B. cinerea & 96 & 98 & 89 & 66 & 51 & 0.34 & 47.9 \\
\hline M. mali & 100 & 100 & 100 & 89 & 83 & $<0.4$ & 11.6 \\
\hline R. solani & 90 & 93 & 93 & 84 & 73 & $<0.4$ & 30.2 \\
\hline S. $^{b)}$ sclerotiorum & 100 & 100 & 100 & 94 & 73 & $<0.4$ & 7.3 \\
\hline
\end{tabular}

a) Means $(n=3) .{ }^{b)}$ Sclerotinia.

Agar media: potato dextrose agar (PDA, Kyokuto Pharmaceutical Industrial Co., Ltd., Tokyo, Japan), Incubation condition: 4 days at $20^{\circ} \mathrm{C}$.

Table 2. Fungicidal activity of penthiopyrad against various pathogens (Spore germination inhibition test)

\begin{tabular}{|c|c|c|c|c|c|c|}
\hline \multirow{3}{*}{$\begin{array}{l}\text { Name of } \\
\text { pathogen }\end{array}$} & \multicolumn{4}{|c|}{ \% Inhibition of germination ${ }^{a)}$} & \multirow{3}{*}{$\mathrm{EC}_{50}(\mathrm{ppm})$} & \multirow{3}{*}{ MIC (ppm) } \\
\hline & \multicolumn{4}{|c|}{ Concentration of penthiopyrad (ppm) } & & \\
\hline & 50 & 10 & 2 & 0.4 & & \\
\hline B. cinerea & 100 & 100 & 100 & 89 & $<0.4$ & 1.4 \\
\hline F. fulva & 100 & 100 & 97 & 60 & $<0.4$ & 6.4 \\
\hline$P$. recondita & 100 & 100 & 94 & 88 & $<0.4$ & 10.0 \\
\hline S. ${ }^{b)}$ sclerotiorum & 100 & 96 & 93 & 70 & $<0.4$ & 10.4 \\
\hline S. ${ }^{c)}$ cucurbitae & 100 & 97 & 74 & 31 & 0.9 & 9.8 \\
\hline V. nashicola & 100 & 97 & 78 & 4 & 1.4 & 8.1 \\
\hline
\end{tabular}

${ }^{a)}$ Means $(n=3) .{ }^{b)}$ Sclerotinia. ${ }^{c)}$ Sphaerotheca. 


\section{Spore germination inhibition test}

$\mathrm{EC}_{50}$ values of penthiopyrad were less than $0.4 \mathrm{ppm}$ to $B$. cinerea, F. fulva, Puccinia recondita, and Sclerotinia sclerotiorum, $0.9 \mathrm{ppm}$ to Sphaerotheca cucurbitae, and $1.4 \mathrm{ppm}$ to V. nashicola. Penthiopyrad showed a strong inhibition effect on the spore germination of various plant pathogens tested as compared with the results of the mycelial growth inhibition test.

Especially in case of $B$. cinerea, penthiopyrad showed significant inhibiting activity on spore germination rather than on mycelial growth. In the case of field application, penthiopyrad showed high preventive effects rather than curative effects against gray mold caused by $B$. cinerea. The strong inhibition effect of penthiopyrad on spore germination seems to be one of the main reasons preventive application brings better gray mold control than curative application.

3. Pot tests

To obtain more than about $80 \%$ of the control effect in pot tests, penthiopyrad needs $25 \mathrm{ppm}$ to tomato leaf mold, $12.5 \mathrm{ppm}$ to rice blast, $6.25 \mathrm{ppm}$ to cucumber powdery mildew and kidney bean gray mold, and $1.56 \mathrm{ppm}$ to wheat brown rust. The results of the pot tests with various kinds of diseases showed that penthiopyrad has a broad spectrum of fungicidal activity. Especially against wheat brown rust, cucumber powdery mildew, and bean gray mold, penthiopyrad was significantly effective even at low doses. No phytotoxicity could be observed in all the tested plants at any dosage tested.

4. Growth inhibition tests for chemical resistant strains of gray mold fungus, B. cinerea, and powdery mildew fungus, Sphaerotheca cucurbitae

4.1. Agar plate dilution test for gray mold fungus, B. cinerea Various kinds of resistant strains in B. cinerea are classified by their performance against benzimidazole compounds, dicarboximide compounds, and diethofencarb as described in section 5.1, Materials and Methods. Penthiopyrad showed a 100\% control effect at 5 ppm against all the kinds of resistant type strains tested as susceptible strains.

Table 3. Control efficacy of penthiopyrad by preventive application in pot test

\begin{tabular}{|c|c|c|c|c|c|c|c|c|c|c|}
\hline \multirow{3}{*}{ Name of disease } & \multicolumn{10}{|c|}{ Control efficacy $(\%)^{a)}$} \\
\hline & \multicolumn{10}{|c|}{ Concentration of penthiopyrad (ppm) } \\
\hline & 200 & 125 & 100 & 62.5 & 50 & 31.2 & 25 & 12.5 & 6.25 & 1.56 \\
\hline Rice blast & 100 & N.T. ${ }^{b)}$ & 100 & N.T. & 93 & N.T. & N.T. & 85 & N.T. & N.T. \\
\hline Tomato leaf mold & 100 & N.T. & 100 & N.T. & 100 & N.T. & 88 & N.T. & N.T. & N.T. \\
\hline Wheat brown rust & N.T. & N.T. & 100 & N.T. & N.T. & N.T. & 100 & N.T. & 100 & 79 \\
\hline Cucumber powdery mildew & N.T. & N.T. & 100 & N.T. & N.T. & N.T. & 100 & N.T. & 78 & 60 \\
\hline Kidny bean gray mold & N.T. & 100 & N.T. & 100 & N.T. & 99 & N.T. & 95 & 86 & N.T \\
\hline
\end{tabular}

${ }^{a)}$ Means $(n=3){ }^{b}$ N.T.: not tested.

Inoculum: Rice blast (Pyricularia oryzae, MCAG stock culture No. 40061), tomato leaf mold (Fulvia fulva, MCAG stock culture No. 40331), wheat brown rust (Puccinia recondita, maintained on the leaves of wheat in greenhouse), cucumber powdery mildew (Sphaerotheca cucurbitae, maintained on the leaves of cucumber in greenhouse), kidny bean gray mold (Botrytis cinerea, MCAG stock culture No. 40212).

Cultivated variety of crops: Rice (Oryza sativa cv. Tsukimimochi), tomato (Solanum lycopersicum cv. Sekaiichi), wheat (Tricicum aestivum cv. Norin No. 61), cucumber (Cucumis sativa cv. Sagamihanjiro), kidny bean (Phaseolus vulgaris cv. Greentop).

Condition of incubation: Rice blast (at $25^{\circ} \mathrm{C}$ for 7 days), tomato leaf mold (in a greenhouse at $22^{\circ} \mathrm{C}$ for 2 weeks), wheat brown rust (kept in a plastic bag

to maintain dark and high humidity at $4^{\circ} \mathrm{C}$ for 2 days followed by incubation in a greenhouse for 8 days), cucumber powdery mildew (in a greenhouse for 7 days), kidny bean gray mold (at $20^{\circ} \mathrm{C}$ for 4 days).

Table 4. Fungicidal activity of penthiopyrad against chemical resistant strains of $B$. cinerea

\begin{tabular}{|c|c|c|c|c|c|c|c|}
\hline \multicolumn{3}{|c|}{ Variety of resistant strains against } & \multicolumn{5}{|c|}{ Tested compounds } \\
\hline Benzimidazole & Dicarboximide & Diethofencarb & Penthiopyrad & $\begin{array}{c}\text { Thiophanate- } \\
\text { methyl }\end{array}$ & Procymidone & Diethofencarb & Mepanipyrim \\
\hline Susceptible & Moderate R. & High R. $\left.{ }^{a}\right)$ & $* * c)$ & $* *$ & - & - & N.T. \\
\hline Moderate R. & Moderate R. & High R. & $* *$ & * & $*$ & - & N.T. \\
\hline High R. & Susceptible & Susceptible & $* *$ & - & $* *$ & $* *$ & N.T. \\
\hline High R. & Moderate R. & Weak R & $* *$ & - & - & $*$ & N.T. \\
\hline High R. & Moderate R. & High R. & ** & - & - & - & N.T. \\
\hline $\mathrm{RAP}^{b)}$ & & & $* *$ & N.T. & N.T. & N.T. & - \\
\hline
\end{tabular}

${ }^{a)}$ R.: Resistant. ${ }^{b)}$ RAP: Resistance to anilinopyrimidine compound. ${ }^{c} * *: 100 \%$ control at 5 ppm of penthiopyrad and procymidone, 100 ppm of thiophanate-methyl, and $10 \mathrm{ppm}$ of diethofencarb. *: between 60 and $99 \%$ control at $100 \mathrm{ppm}$ of thiophanate-methyl, $5 \mathrm{ppm}$ of procymidone, and $10 \mathrm{ppm}$ of diethofencarb. - : less than $59 \%$ control at $100 \mathrm{ppm}$ of thiophanate-methyl, $5 \mathrm{ppm}$ of procymidone, and $10 \mathrm{ppm}$ of diethofencarb, N.T.: not tested. 
Table 5. Control efficacy of penthiopyrad against chemical resistant strains of powdery mildew fungus, Sphaerotheca cucurbitae

\begin{tabular}{|c|c|c|c|c|c|c|c|c|}
\hline \multirow{3}{*}{ Test compound } & \multicolumn{4}{|c|}{ \% Control against $S-S^{a)}$} & \multicolumn{4}{|c|}{$\%$ Control against $\mathrm{R}-\mathrm{S}^{b)}$} \\
\hline & \multicolumn{4}{|c|}{ Conc. of penthiopyrad (ppm) } & \multicolumn{4}{|c|}{ Conc. of penthiopyrad (ppm) } \\
\hline & 100 & 10 & 1 & 0.1 & 100 & 10 & 1 & 0.1 \\
\hline Penthiopyrad & 100 & 100 & 29 & 0 & 100 & 100 & 53 & 0 \\
\hline Azoxystrobin & 100 & 100 & 100 & 100 & 80 & 13 & 0 & 0 \\
\hline
\end{tabular}

${ }^{a)} \mathrm{S}-\mathrm{S}$ : susceptible strain to strobilurin compound. ${ }^{b} \mathrm{R}-\mathrm{S}$ : resistant strain to strobilurin compound.

4.2. Leaf disk test for cucumber powdery mildew fungus, Sphaerotheca cucurbitae

Azoxystrobin usually showed excellent control effects on cucumber powdery mildew, and $0.1 \mathrm{ppm}$ of azoxystrobin could perfectly control it. However, the \% control against the resistant strain was 80 at $100 \mathrm{ppm}$. On the other hand, penthiopyrad showed equivalent efficacy against the strobilurin-resistant strain as against the susceptible strain. Penthiopyrad showed a perfect control effect at $10 \mathrm{ppm}$ against both susceptible and resistant strains of strobilurin.

Penthiopyrad shows a broad range antifungal spectrum against various plant pathogens and significant effects for controlling a wide range of plant diseases including gray mold, powdery mildew, and tomato leaf mold. This means it may be possible to control three diseases with one application of penthiopyrad. In addition to the wide fungicidal spectrum, penthiopyrad has no cross-resistance with benzimidazoles, dicarboximides, anilinopyrimidines, DMIs, and strobilurin compounds. It is concluded that penthiopyrad is a superior fungicide that has a very wide range of fungicidal activity, especially on spore germination, and a broad spectrum in disease control. As described in the result of the spore germination inhibition test, penthiopyrad is recommended for use in preventive application to obtain the best disease control performance.

\section{References}

1) M. Kulka and B. von Schmeling: "Modern Selective FungicidesProperties, Applications, Mechanisms of Action-," ed. by H. Lyr, 2nd Ed., Gustav Fischer Verlag, Germany and New York, Chap. 8, pp. 133-147, 1995.

2) L. V. Edgington and G. L. Barron: Phytopathology 57, 1256-1257 (1967).

3) M. Oda, N. Sasaki, T. Sasaki, N. Nonaka, K. Yamagishi and H. Tomita: J. Pestic. Sci. 17, 91-98 (1992).

4) K. Tomiya and Y. Yanase: The BCPC International Congress Crop Science \& Technology, 2A-9, pp. 99-104, 2003.

5) H. Katsuta, Y. Yoshikawa, K. Tomiya, H. Kawashima, O. Takahashi, T. Kitashima, S. Inami, Y. Yanase and J. Kishi: Abstr. 29th Annu. Meeting Pestic. Sci. Soc. Jpn., p. 55, 2004 (in Japanese).

6) Y. Yanase, T. Akase, J. Kishi, N. Tomura, S. Inami, H. Shimotori, H. Katsuta, K. Tomiya and Y. Yoshikawa: Abstr. 29th Annu. Meeting Pestic. Sci. Soc. Jpn., p. 56, 2004 (in Japanese).

7) Y. Yanase, Y. Yoshikawa, J. Kishi and H. Katsuta: 11th IUPAC International Congress of Pesticide Chemistry, 31, p. 296 (2006).

8) Y. Yoshikawa, H. Katsuta, J. Kishi and Y. Yanase: J. Pestic. Sci. 36, 347-356 (2011). 